\title{
Design of Virtual Street Platform Based On 3G Network
}

\author{
Zhang Li, Feng Shi-long \\ College of Software Beijing University of Technology, Beijing 100124, China \\ \{fslong0459\&zl_hlj\}@126.com
}

\begin{abstract}
In order to meet development tendency of mobile internet and the needs of LBS(location-based services) applications, we carried out a research of framework called virtual street platform(VSP), which used GPS, P2P and IP communitaction.

Index Terms: 3G; LBS; mobiles P2P;mobiles IP

\section{Introduction}

3G network users has been more than 200 million in China, Increased 88\% than 2011. [1][2].Better services and applications based on intelligent mobile terminal are demanded. Now a growing number of intelligent terminal were equipped with GPS module which can provide hardware foundation of location-based services.

The VSP closely relates to the user location.The platform allows users entered a street to perceive the surrounding environment with the device and interact with the surrounding environment and people.
\end{abstract}

\section{Design of VSP}

\subsection{Architecture of VSP}

In our design concept, we hope that the VSP is so flexibility and scalability that it can be established any place any time. But the number of clients and the avility of providing services are limited by server processing power and resource constraints in $\mathrm{C} / \mathrm{S}$ architecture system. For the above reason, we eventually determined to use hybrid peer-to-peer architecture which refered to Jxta-jxme and Skype[3].The platform consists of a central server, super nodes(determined by election within the scope of each street) and user nodes, shown in Figure 1

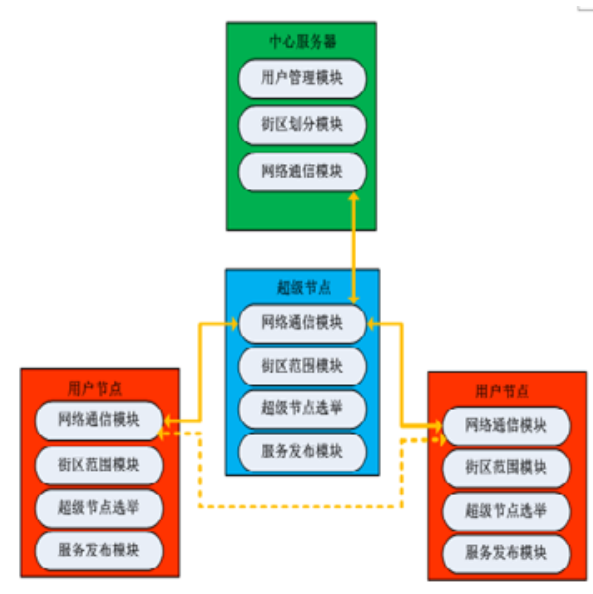

Fig.1 Network architecture of VSP
The actual deployment is shown in Figure 2.

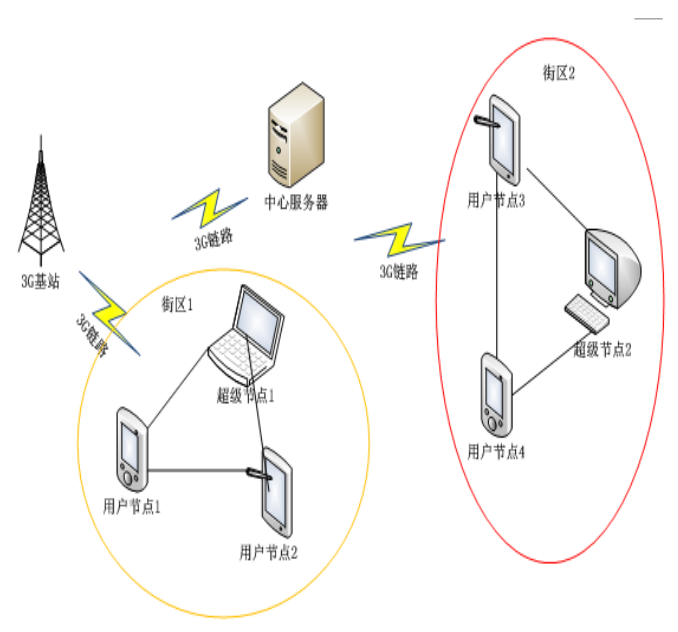

Fig.2 The actual deployment of VSP

\subsection{Design of VSP Network communication}

Network communication of VSP includes four cases: between the central server and the super node, between the center server and the user node, between the super node and the user node, among the user nodes. The first three cases can be classified as $\mathrm{C} / \mathrm{S}$ mode of communication because at least one of the two communication sides has fixed public IP, and technology has been very mature, they will not be the discussed.

We need to focus on the fourth case and discuss it in detail, the communication among the user nodes. In this communication case we need to face the two issues previously mentioned: the existence of dynamic IP and NAT. Dynamic IP problem found after the author's experiment is that local IP and public IP assigned by ISP of the mobile terminal under the 3G network change dynamically, which caused the difficulties of the packet exchange among the user nodes.

According to the mapping ways NAT can be divided into two types: cone NAT and symmetric NAT. The existence of NAT increased communication difficulty, so it is necessary to consider how to traverse NAT. Relative to the cone NAT, symmetric NAT is more difficult to traverse. And the most difficult of the traversal conditions are both communication terminals located in symmetric NAT, but this is precisely the most common communication among the $3 \mathrm{G}$ network mobile 
terminal. So the focus of our study about NAT traversal is communication both sides in symmetric NAT.

NAT traversal preferred solution is ICE which integrated STUN, TURN and other protocols. On the basis of the above discussion, we draw on the ICE principle, and take into account the the VSP requirements and network architecture design, and finally determine communication among user nodes need the assit of super node with fixed public IP. The detailed process is described below.

Assuming that the user node $\mathrm{A}$ initiate a communication to the user node $\mathrm{B}$, and the two user nodes have successful log in VSP. User node A send a message to super node of the street, the message including the destination node (the user node $\mathrm{B}$ ), the message body (specifically the contents of the message, such as "Who are you?") And message ID (for the acknowledgment of message communication); And the super node is temporarily saved to the message, when the super node get heartbeat of node $B$, the message is sended to node $B$ as feedback of heartbeat; Then $B$ receive the message and send confirmation message (includes message ID) to the super node; The super the nodes temporarily stored it and send confirmation message to the user node $\mathrm{A}$ as the feedback of heartbeat of node $A$. Nodes $A$ receive the confirmation message, and return the message to the super node "communication was successful", finally the super node delete data corresponding message ID. If node A does not receive feedback messages, it means the message is failed to send and resend the message again.

Since the communication between two user nodes using commonly short connection, the presence of user node dynamic IP and NAT in 3G networks, and the initiator of each time communication is always the user node (client), platform determine to use HTTP protocol which is simple, fast and good at NAT traversal as the communication protocol between two user nodes. And communication data format use Json. HTTP protocol system has the agent (proxy in the client) and the gateway (Gateway, located on the server), so under normal circumstances it can traverse most of NAT. Considering to reduce the complexity of the platform,we determine to use that communication in the other three cases mentioned previously.

\section{Design of Every Part of the Platform}

\subsection{Central Server}

The central server has a fixed public IP. It includes network communication sub-module, user management submodule, street divition sub-modules and services published sub-module.

\subsubsection{Network Communication Sub-Module}

This sub-module is responsible for the VSP network communication, the server must have a public IP. This submodule determines the communication protocols and communication formats of the whole platform, such as: all messages need the string of "VirtualStreetData" as the message header; It is also responsible for the responding to requests of super nodes and user nodes.

\subsubsection{User Management Sub-Module}

This module is responsible for the registration and login of the user, and saving the user's registration information and basic personal information and records including the login location information, it is also responsible for the user identity verification when the user login the platform. After successful login, the central server will send the information(including access and port) of the super node of the street which the user belongs to. It also determine the election rules of alternative super node to ensure that there are both a super node and an alternative super node in each street of platform.

\subsubsection{Street Divition Sub-Module}

The module is responsible for the scope of each virtual street and judgment of new login user nodes belong to which street, this procedure is based on the geographical space. The principle of dividing street of platform is based on Google's location services, and the list of common virtual street is stored in the central server, and update regularly. In addition, the central server should notify street division to the super node. We need to consider the street division in various kinds of actual cases.

\subsection{Super Node}

Super node theoretically should have a public IP. Super node function modules include network communications submodule, street scope sub-module, super nodes election submodule and services released sub-module.

\subsubsection{Network Communication Sub-Module}

This module is responsible for the communication between the super node and the central server and the communication between the super node and the user node. Communication between super node and the central server includes two aspects, one is super nodes sending heartbeat messages every 10 minutes to the central server; The other is when a user node leave the street the super node in charge of, the super node needs to report the username to the central server in time. Tasks of the communication between the super node and local user nodes include the response to the user's heartbeat, maintaining, updating and broadcasting the local user list, user nodes IP and port, transmitting the message and assisting to establish $\mathrm{P} 2 \mathrm{P}$ communication connection between user nodes.

\subsubsection{Street Scope Sub-Module}

This module is responsible for analizing the scope information of the streets from the central server, and determining the user nodes are in and out of the street area by the heartbeat.

\subsubsection{Super Node Election Sub-Module}

This module is responsible for electing a node as the alternative super node by the comparison of the IP address, the comparison of the hardware configuration and considering the the super node standard provided by the central server. Then, the super node sends the list of users to the alternative super node, and sends the IP address, port number and other 
information of the alternative super node to the local user nodes and the centeral server.

\subsubsection{Service Released Sub-Module}

This module is responsible for the release of server service for all users of the platform, including online games, ebooks and other leisure entertainment.

\subsection{User Node}

The function modules of user node include network communication sub-module, geolocation and terminal configuration scanning sub-module and service discovery submodule.

\subsubsection{Network Communication Sub-Module}

Network communication sub-module is responsible for the communication between the user node and the central server, the communication between the user node and super node and the communication between different user node,and send the heartbeat to super node every 30 second.

In addition, the module need to implement the interface of communication with services(we focus on online game)that platform released, in order to achieve that users can enjoy the new services of the platform in real-time.

\subsubsection{Geographical Location and Terminal Configuration Scan Sub-Module}

This module is responsible for the real-time geographical location of the user node terminal by GPS system, and reporting this information to the central server, then reporting this information to the super node of the street which the user node belongs by the heartbeat information. In addition, this module is also responsible for the scanning of terminal hardware configuration and reporting the information to the super node, which is used for election of the alternative super node of the street.

\subsubsection{Service Discovery Sub-Module}

This module is responsible to check whether there are the newly released server services, such as online games 。

\subsection{VSP Implementation}

The development of the platform server (including servers and super nodes) use JavaEE technology. We use Struts + Spring + Hibernate in the middle layer and lightweight MySQL database in saving nodes information.We determine to use the Android for the client.

\section{Decription of Running Effect}

After the completion of the design and implementation of VSP, let's have the actual deployment of the platform based on 3G network and run it. The deployment environment is as follows:

1. A central server (HP DC7900 desktop, Win7 32-bit operating system);

2. Super node (Toshiba L600-58W notebook, Win 7 32-bit operating system);
3. User node A(Samsung I9250 Android operating system, Unicom's WCDMA 3G network), the user node B (HTC Z710e, Unicom's WCDMA 3 G network);

4. Third-party software support: Oray(kind of DDNS).

The running process is as follows:

1. Start the central server;

2. Start street super node and register,then login;

3. User node A register and login;

4. User node B register and login;

Center server and super node has not yet developed the user interface, they have the same working mechanism which is running in the background and transparent to the user. They have the same running effect. Figure 3 shows the central server running in debugging environment.

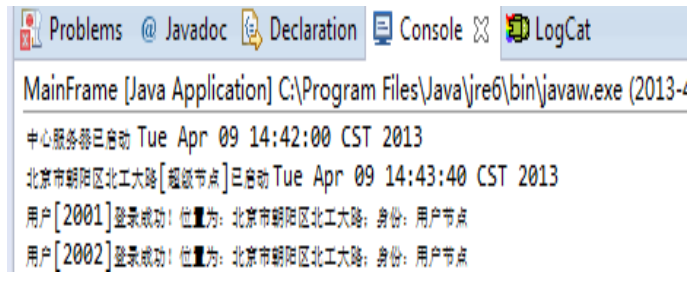

Fig.3 The effect of running central server

The function provided by the VSP mainly include street divition, chat, inquiries, announcements, service releasing and discovery. The following is the results of running the functions the platform provides.

The user "fireworks" (platform nickname) in Beijing University of Technology login platform, located by the local GPS, the location information is analysed by Google geographic service, and the analytical results (Beijing, Chaoyang District,BJUT Road) are sended to the central server. Then the central server sends the super node address (it is the Oray domain name in lab environment)and port information to the user node. The user node signs itself to the super node and gets user nodes list in the same street, shown as right one of Figure 5(The right one), the user "strawberry sweet " in the figure is in the same street (BJUT Road) with user "fireworks".

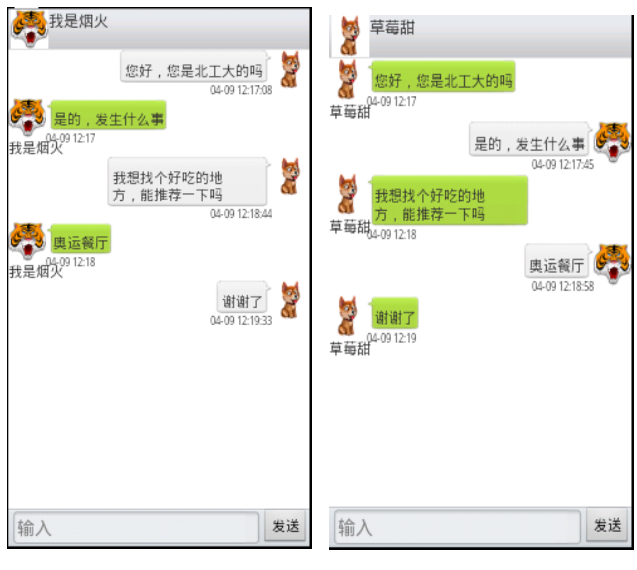

Fig.4 Chatting 
Chat, inquiries and announcements are the main communication mechanism of the VSP. The user "strawberry sweet" inquires of the user "fireworks" about the street information. Basic communication between nodes is shown in Figure 4; Left one of figure 5 is the effect of street announcement.

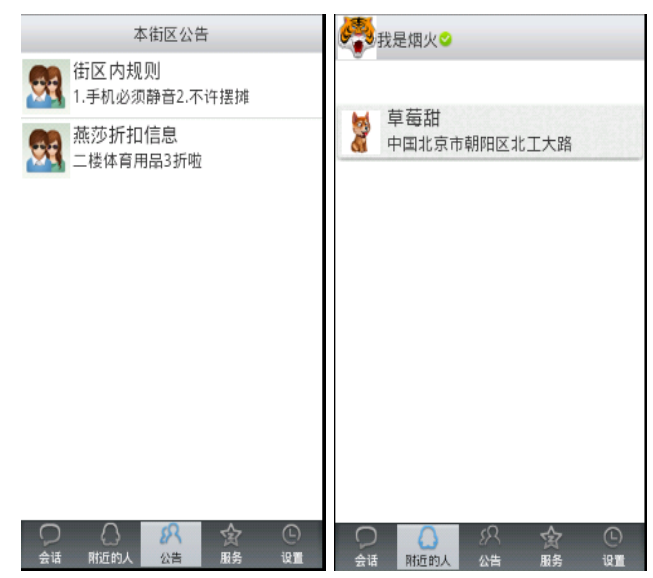

Fig.5 Anouncement and user list

Street service release and discovery. Street users can release their local services (e-books, online games, etc.) on the VSP. We take online game for example, there is a chess game, and the size of the installation file is $150 \mathrm{k}$. At first user "fireworks" upload the installation files to the super node. Announcement of the super node notice the information to other local user "strawberry sweet", so the services discovery of platform is implemented; Then user "strawberry sweet" downloads the game from the super node and deploys in the local device. From the user "fireworks" release to user "strawberry sweet" successful deployment, the entire process takes about 50 seconds. User "strawberries sweet" start the game, she starts the "Round" first, the game data is enveloped into the format defined by the network communication submodule the of user node of the platform, via the service interface of the sub-module complete the interaction of game data, the game data is transmitted to another a user "firework", the data transfer takes about 7 seconds and has a good user experience in real-time performance.

\section{Summary}

On the basis of many domestic research and foreign research, the VSP designed in accordance with the program described in this article can effectively reduce the burden on the central server.The VSP based on 3G network we had finished the implementation, which used peer-to-peer network architecture and space positioning technology, can effectively help users on platform to do part of social activities with other users in the same street which is classified by platform, activities include making friends, chat, online games, and sharing of resources,additionally strong position correlation difference to many other platforms also improve the level of trust and intimacy between users. We got some results in overcoming the particularity of IP communications among mobile terminal based on 3G network, such as successful traversal of the various types of NAT (3G network mobile terminal is generally symmetric NAT) and solution of the terminal dynamic IP communication problems.Platform release of games and other services, just released at the super nodes, then the user nodes can download and install it. Game data are encapsulated into Json and the interacted among the user nodes by the network communication sub-module of each part of platform.

There are still some shortcomings in VSP.Taking into account the burden on the server and super nodes, we design the heartbeat interval for a long time, thus affecting the realtime capability of platform communication, so we need to improve hardware performance of the central server and super node, and try to find a solution of lighter burden on the central server and better real-time performance.

\section{References}

[1] Chen Zhang, Jinghua Huang, Jian Chen, Research on Adoption of Mobile Virtual Community in China and Korea, 2010 Ninth International Conference on Mobile Business / 2010 Ninth Global Mobility Roundtable, Pages:220-227.

[2] Chung-Pyo Hong, Eo-Hyung Lee, and Shin-Dug Kim, An Efficient Scheme to Construct Virtual Community for Multimedia Content Sharing based on Profile in a Ubiquitous Computing Environment, 2009 Fifth International Joint Conference on INC, IMS and IDC, Page:1271-1276.

[3] Zhang Chun-hong, Comprehensive Analysis of P2P Technology,:Post\&Telecom Press, pp. 22-43. 